\title{
Critical exponent for the quantum Hall transition
}

\author{
Keith Slevin \\ Department of Physics, Graduate School of Science, Osaka University, \\ 1-1 Machikaneyama, Toyonaka, Osaka 560-0043, Japan \\ Tomi Ohtsuki \\ Department of Physics, Sophia University, Kioi-cho 7-1, Chiyoda-ku, Tokyo 102-8554, Japan
}

(Dated: October 22, 2018)

\begin{abstract}
We report an estimate $\nu=2.593[2.587,2.598]$ of the critical exponent of the Chalker-Coddington model of the integer quantum Hall effect that is significantly larger than previous numerical estimates and in disagreement with experiment. This suggests that models of non-interacting electrons cannot explain the critical phenomena of the integer quantum Hall effect.
\end{abstract}

The most important characteristics of a continuous phase transition are its critical exponents. The critical exponents are a quantitative characteristic of the critical fixed point. Agreement of theory with experiment implies that the critical fixed point studied theoretically is the same fixed point observed in the experiment. For the integer quantum Hall transition [1] the value of the critical exponent $\nu$ that describes the divergence of the localization length $\xi$ of the electrons has now been measured to an accuracy of a few percent [2, 3], so comparison with experiment has become a stringent test of theory. One of the most surprising aspects of the integer quantum Hall transition is the agreement between experiment and theory [4, 5, 66, 7] concerning the value of the critical exponent. The agreement is surprising because the Coulomb interaction between the electrons is not included in the theoretical models [8, 9, 10]. It suggests that the Coulomb interaction is not relevant at the integer quantum Hall critical point. Yet, this is immediately contradicted when we look at the dynamical critical exponent $z$. For models of non-interacting electrons $z$ is exactly 2 [11], whereas the measured value is $\approx 1[3]$.

We show in this paper that previous theoretical work has significantly underestimated the critical exponent $\nu$ for non-interacting electrons and that there is in fact a clear disagreement with experiment. This suggests that models of non-interacting electrons cannot explain the critical phenomena of the integer quantum Hall effect.

Non-interacting electron models of the integer quantum Hall transition are also of interest in their own right. In particular, though the critical field theory for such models is as yet unknown 12, it has been speculated that it should have conformal invariance [13]. If so, a scaling relation follows [14], which we test by comparing recent multi-fractal analyses [15, 16] with our work.

The integer quantum Hall effect [1] occurs in two dimensional electron gases that are subject to a large perpendicular magnetic field. The application of the field results in the quantization of the kinetic energy of the electrons and the formation of Landau levels. Impurity scattering causes the Landau levels to broaden into Landau bands. The states at the center of the Landau band are critical while other states are localized. A quantum Hall transition between quantized values of the Hall resistance occurs whenever the Fermi energy passes through the center of a Landau band [6, 17]. The critical exponent $\nu$ describes the divergence of the localization length

$$
\xi \sim\left|x-x_{\mathrm{c}}\right|^{-\nu},
$$

near the critical energy. Here, $x$ can be any control parameter, for example the Fermi energy, that drives the two dimensional electron gas though the transition at $x_{c}$. In common with other continuous phase transitions, the value of the critical exponent is expected to exhibit a high degree of universality [13].

We have performed a finite size scaling (FSS) analysis of the quantum Hall transition in the ChalkerCoddington network model [10, 18]. In this model the motion of the electron in a random potential and quantizing magnetic field is replaced by the transmission of an electron through a network of links and nodes. The links describe electron motion along lines of constant potential and the nodes describe the scattering of electrons at saddle points of the potential. The Coulomb interaction between the electrons is ignored.

We use the transfer matrix method to estimate the smallest positive Lyapunov exponent of a very long network consisting of $L$ layers. Each layer consists of two sub-layers: one a transverse array of $N$ nodes of type A and the other a transverse array of $N$ nodes of type B. A transfer matrix $T_{l}$ of size $2 N \times 2 N$ relates the $N$ rightgoing and $N$ left-going flux amplitudes at the left of the layer to the similar quantities at the right of the layer

$$
\left(\begin{array}{c}
a^{\prime}{ }_{1} \\
b^{\prime}{ }_{1} \\
\vdots \\
a^{\prime}{ }_{N} \\
b^{\prime}{ }_{N}
\end{array}\right)=T_{l}\left(\begin{array}{c}
a_{1} \\
b_{1} \\
\vdots \\
a_{N} \\
b_{N}
\end{array}\right)
$$

The explicit form of the transfer matrix is

$$
T_{l}=B V_{l} A U_{l}
$$


The scattering at nodes of type $\mathrm{A}$ is described by the matrix

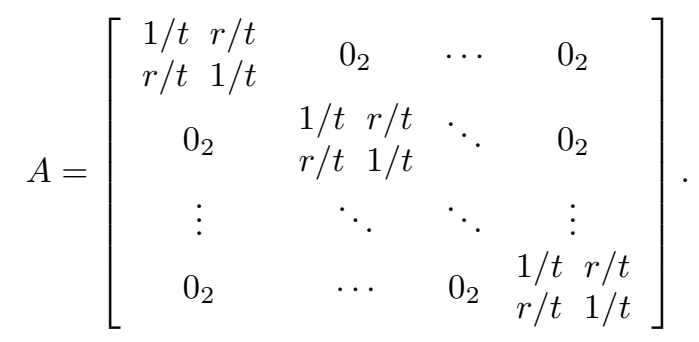

Here, $0_{2}$ is the $2 \times 2$ zero matrix, $t$ is the probability amplitude with which current from the left is transmitted to the right, while $r$ is the amplitude with which current from the left is reflected back to the left. Nodes of type $\mathrm{B}$ are obtained from nodes of type A by a rotation of $90^{\circ}$. Imposing periodic boundary condition in the transverse direction we then have

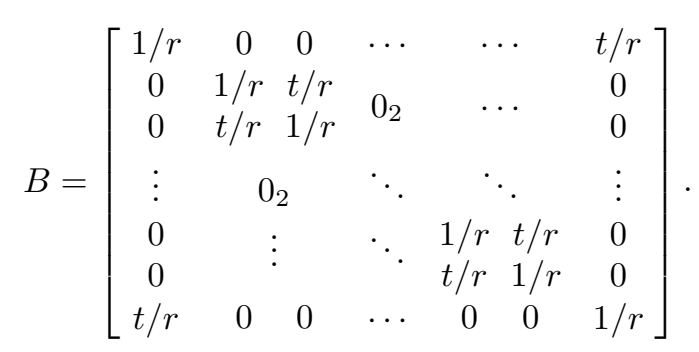

The transmission and reflection amplitudes are parameterized by the parameter $x$ according to

$$
t=(\exp (2 x)+1)^{-1 / 2}, r=\sqrt{1-t^{2}}
$$

The parameter $x$ may be interpreted as the energy of the electron measured from the Landau band center scaled by the Landau band width. The matrix $U_{l}$ is a diagonal matrix with elements

$$
\left(U_{l}\right)_{m, n}=\delta_{m, n} \exp \left(\mathrm{i} \varphi_{l, m}\right)
$$

and similarly $V_{l}$ is a diagonal matrix with elements

$$
\left(V_{l}\right)_{m, n}=\delta_{m, n} \exp \left(\mathrm{i} \varphi_{l, m}^{\prime}\right)
$$

Since the distances between the nodes are random, we suppose that the phases are independently and uniformly distributed on $[0,2 \pi)$. (These random numbers were generated using the routine ran described in Chapter B7 of [19].)

The transfer matrix of the network model is equal to the product of the transfer matrices of the layers

$$
T=\prod_{l=1}^{L} T_{l}
$$

From this matrix we can define a hermitian matrix $\Omega$ by

$$
\Omega=\ln \left(T^{\dagger} T\right)
$$

As a consequence of the conservation of flux the eigenvalues of this matrix occur in pairs of opposites sign

$$
\left\{+\nu_{1}, \cdots,+\nu_{N},-\nu_{N}, \cdots,-\nu_{1}\right\}, \nu_{1}>\cdots>\nu_{N}>0 .
$$

We are interested in the smallest positive Lyapunov exponents which is obtained in the following limiting procedure

$$
\gamma=\lim _{L \rightarrow \infty} \frac{\nu_{N}}{2 L}
$$

The value obtained is independent of the particular sequence of transfer matrices in the transfer matrix product for almost all sequences. Truncation of the transfer matrix multiplication at a large but finite $L$ yields an estimate of the Lyapunov exponent with a known precision. Repeated QR factorizations are needed to avoid a loss of precision due to round off error [20].

To analyze the simulation data we assume that the dimensionless quantity

$$
\Gamma=\gamma N
$$

obeys an FSS law

$$
\Gamma=F_{0}\left(N^{\alpha}\left(x-x_{c}\right)\right)
$$

Here, $\alpha$ is the reciprocal of the critical exponent $\nu$. In practice, the data deviate from this law. This is taken into account by including corrections to scaling that arise from non-linearity of the scaling variables and irrelevant scaling variables. In previous work 21] on the 3D Anderson model, we have found that the fitting formula

$$
\Gamma=F_{0}\left(N^{\alpha} u_{0}\right)+F_{1}\left(N^{\alpha} u_{0}\right) N^{y} u_{1},
$$

works well. Here, $u_{0} \equiv u_{0}(x)$ and $u_{1} \equiv u_{1}(x)$ are, respectively, relevant and irrelevant scaling variables, and $F_{0}$ and $F_{1}$ are corresponding scaling functions. The exponent $y$ associated with the irrelevant variable is negative, $y<0$. The relevant variable is zero at the critical point. For the Chalker-Coddington model the critical point is known to be exactly $x_{c}=0$ and does not need to be found by fitting the numerical data, so $u_{0}(0)=0$.

When periodic boundary conditions are imposed, swapping $t$ and $r$, and translating the network so that the $\mathrm{A}$ and $\mathrm{B}$ nodes are interchanged yields an identical network. As a result of this symmetry $\Gamma$ is an even function of $x$. (Note that this holds only for periodic boundary conditions.) To reflects this, we make $F_{0}, F_{1}$ and $u_{1}$ even, and $u_{0}$ odd, functions. The condition on $u_{0}$ follows because the relevant variable is zero at the critical point.

We are able to fit our numerical data to (1) provided that data for smaller system sizes $(N<16)$ are excluded. All the functions appearing in (1) are expanded in Taylor series. The results of the fit are summarised in Table [1] The data and the fit are plotted in Figs. 1 and 2 . 


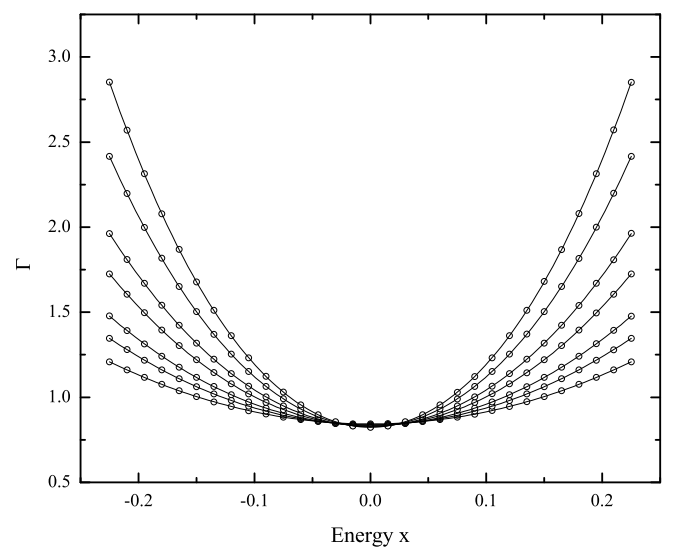

FIG. 1: Simulation data and FSS fit for the ChalkerCoddington model. The different lines correspond to different numbers of nodes $N=16,24,32,48,64,96,128$.

$\begin{array}{ccc} & \text { estimate } & 95 \% \text { confidence interval } \\ \alpha & 0.3857 & {[0.3849,0.3866]} \\ \Gamma_{\mathrm{c}} & 0.780 & {[0.767,0.788]} \\ y & -0.17 & {[-0.21,-0.14]}\end{array}$

TABLE I: The least squares fit of the FSS model to the simulation data. The number of data points is $N_{\mathrm{D}}=217$ and the number of parameters is $N_{\mathrm{P}}=9$. The minimum value of $\chi^{2}=199.8$ and the goodness of fit $p=0.6$. The series for $F_{0}, u_{0}, F_{1}$ and $u_{1}$ were truncated at orders $6,3,0$ and 2, respectively.

The confidence intervals have been obtained from Monte Carlo simulation [22].

It is clear from Fig. 2 that corrections to scaling are of the order of a few percent for the smallest $N$. The precision of our data is $0.03 \%$, which requires $L \approx 10^{8} \sim$ $10^{9}$ transfer matrix multiplications to achieve. Such high precision is required because the dependence on energy of $\Gamma$ is very weak near the critical point for the available $N$ and the critical exponent is, in effect, estimated from the variation of the curvature with $N$.

We have tested the stability of this fit when the data are filtered versus the range of $\Gamma$ (Table III), the range of $N$ (Table III) and also checked for stability against increases in the the order of the various Taylor expansions (Table IV). In all cases there is a large overlap of the confidence intervals with the fit in Table [.

Our FSS analysis yields the following best fit value and $95 \%$ confidence interval for the critical exponent

$$
\nu=2.593[2.587,2.598] \text {. }
$$

This result is consistent with but also a considerable improvement on the original estimate $\nu=2.5 \pm .5$ [10] of Chalker and Coddington.

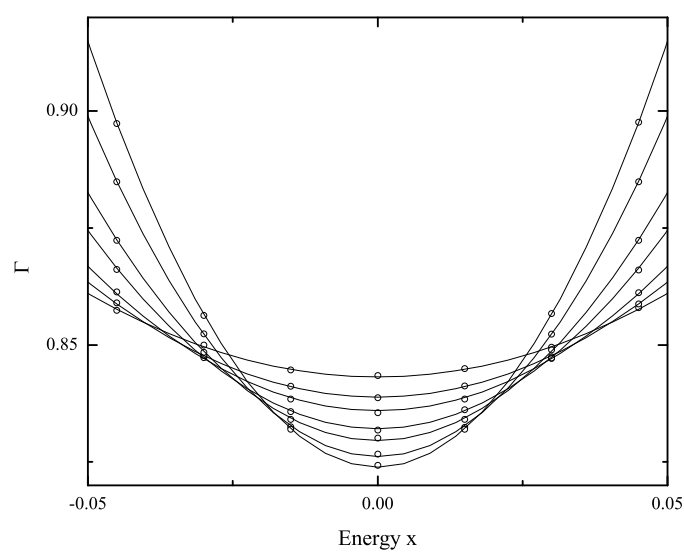

FIG. 2: The same as Figure 2 but with the focus on data near the critical point so as to make the existence of corrections to scaling due to an irrelevant scaling variable evident.

$$
\begin{array}{lllll}
\Gamma<2.0 & 0.3858 & {[0.3850,0.3867]} & 0.779 & {[0.767,0.788]} \\
\Gamma<1.5 & 0.3855 & {[0.3847,0.3864]} & 0.781 & {[0.769,0.790]} \\
\Gamma<1.0 & 0.3826 & {[0.3796,0.3858]} & 0.789 & {[0.778,0.797]}
\end{array}
$$

TABLE II: The best fit of $\alpha$ and $\Gamma_{c}$ with $95 \%$ confidence intervals. The data have been filtered using the condition on $\Gamma$ at the left.

Our estimate is significantly larger than the oft quoted result of $\nu=2.34 \pm .04$ of Huckestein and Kramer [5] for a random Landau matrix model. This disagreement could be taken as evidence against the universality of the critical exponent. However, we feel that a more likely explanation is that the precision claimed by Huckestein and Kramer is too optimistic. This may be clarified in future work.

Our result is also different from the analytical result $\nu=7 / 3$ of Milnikov and Sokolov [4]. However, this value is not expected to describe the true critical point but an intermediate behavior not too close to the critical point.

Our result also disagrees with $\nu=2.37 \pm 0.02$ of Cain et al. 7 based on a real space renormalization group approach 23]. A more recent calculation [24] on a network with a triangular lattice gave $\nu \approx 2.3 \sim 2.76$. Thus, the precision claimed by Cain et al. may be questionable. Moreover, this approach involves an uncontrolled approximation and so the discrepancy may not be signif-

$$
\begin{array}{lllll}
N \geq 24 & 0.3854 & {[0.3845,0.3864]} & 0.782 & {[0.763,0.794]} \\
N \geq 32 & 0.3849 & {[0.3837,0.3861]} & 0.787 & {[0.768,0.800]}
\end{array}
$$

TABLE III: The best fit of $\alpha$ and $\Gamma_{c}$ with $95 \%$ confidence intervals. The data have been filtered using the condition on $N$ at the left. 
$\begin{array}{lllll}F_{0} & 0.3858 & {[0.3849,0.3866]} & 0.779 & {[0.767,0.788]}\end{array}$

$u_{0} \quad 0.3853[0.3843,0.3864] 0.780[0.767,0.789]$

$F_{1} \quad 0.3828[0.3754,0.3917] 0.780[0.767,0.789]$

$\begin{array}{llll}u_{1} & 0.3857[0.3849,0.3865] & 0.780[0.767,0.788]\end{array}$

TABLE IV: The best fit of $\alpha$ and $\Gamma_{c}$ with $95 \%$ confidence intervals. The order of the expansion of the indicated function has been increased by two compared with TABLE [

icant.

More important than the discrepancy with previous theory is the disagreement of our estimate with recent experiments. Li et al [2, 3] measured $\nu=2.38 \pm .06$ in experiments on GaAs-AlGaAs heterostructures. In these experiments, an exponent $\kappa=1 / \nu z$ was measured. Extraction of the critical exponent $\nu$ requires an independent measurement of the dynamical exponent $z$. Li et al measured $z \approx 1$, but seem to have obtained their result for $\nu$ by supposing $z=1$. An unambiguous comparison with theory will require a more careful consideration of the precision of the estimate of $z$.

In our opinion, the coincidence of previous theoretical and experimental estimates of $\nu$ is not significant. Noninteracting theory predicts $z=2$ in clear disagreement with experiment. Calculations 11] within the HartreeFock approximation suggest that the observed value of $z \approx 1$ may be explained by including the Coulomb interaction. We speculate that the discrepancy in the value of $\nu$ that we report here may also be explained in this way.

Assuming that the critical field theory of the integer quantum Hall effect has conformal invariance, it has been shown that $\Gamma_{c}$ and $\alpha_{0}$ (determined in multi-fractal analysis [15, 16]) are related by [14]

$$
\Gamma_{c}=\pi\left(\alpha_{0}-2\right) \text {. }
$$

Eq. (3) follows from a conformal mapping between the strip used in transfer matrix calculations and the $2 \mathrm{D}$ plane used in multi-fractal analysis. Inserting our result for $\Gamma_{c}$ into this formula we obtain

$$
\alpha_{0}=2.248 \quad[2.244,2.251] \text {. }
$$

This value is not consistent with either $2.2617 \pm 0.0006$ reported in [15] or 2.2596 \pm 0.0004 in [16]. However, we cannot conclude that the critical theory does not have conformal invariance because the leading correction to scaling decays very slowly with $N$, as is indicated by the small value of the irrelevant exponent $y$. This does not affect the estimation of the critical exponent $\nu$ but it does complicate the estimation of $\Gamma_{c}$ and, probably, $\alpha_{0}$.

In conclusion, we report an estimate of the critical exponent $\nu$ of the integer quantum Hall effect that is significantly larger than both previous theoretical estimates and, more importantly, experimentally measured values. We speculate that models of non-interacting electrons cannot explain the critical phenomena of the integer quantum Hall effect. Further work is needed to come to a definite conclusion concerning the conformal invariance of the critical theory of non-interacting electron models of the integer quantum Hall transition.

This work was supported by Grant-in-Aid No. 18540382. We would like to thank H. Obuse, A. Furusaki, F. Evers and A. M. Tsvelik for helpful comments.

[1] K. v. Klitzing, G. Dorda, and M. Pepper, Phys. Rev. Lett. 45, 494 (1980).

[2] W. Li, G. A. Csathy, D. C. Tsui, L. N. Pfeiffer, and K. W. West, Phys. Rev. Lett. 94, 206807 (2005).

[3] W. Li, C. L. Vicente, J. S. Xia, W. Pan, D. C. Tsui, L. N. Pfeiffer, and K. W. West, Phys. Rev. Lett. 102, 216801 (2009).

[4] G. Mil'nikov and I. Sokolov, JETP Lett. 48, 536 (1988).

[5] B. Huckestein and B. Kramer, Phys. Rev. Lett. 64, 1437 (1990).

[6] B. Huckestein, Rev. Mod. Phys. 67, 357 (1995).

[7] P. Cain, R. A. Romer, and M. E. Raikh, Phys. Rev. B 67, 075307 (2003).

[8] L. Schweitzer, B. Kramer, and A. MacKinnon, J. Phys. C 17, 4111 (1984).

[9] T. Ando and H. Aoki, Journal of the Physical Society of Japan 54, 2238 (1985).

[10] J. T. Chalker and P. D. Coddington, J. Phys. C 21, 2665 (1988).

[11] B. Huckestein and M. Backhaus, Phys. Rev. Lett. 82, 5100 (1999).

[12] A. M. Tsvelik, Phys. Rev. B 75, 184201 (2007).

[13] J. L. Cardy, Scaling and renormalization in statistical physics (Cambridge University Press, Cambridge, 1996).

[14] M. Janssen, Fluctuations and localization in mesoscopic electron systems, World Scientific lecture notes in physics ; v. 64 (World Scientific, Singapore, 2001).

[15] H. Obuse, A. R. Subramaniam, A. Furusaki, I. A. Gruzberg, and A. W. W. Ludwig, Phys. Rev. Lett. 101, 116802 (2008).

[16] F. Evers, A. Mildenberger, and A. D. Mirlin, Phys. Rev. Lett. 101, 116803 (2008).

[17] H. Aoki and T. Ando, Solid State Commun. 38, 1079 (1981).

[18] B. Kramer, T. Ohtsuki, and S. Kettemann, Physics Reports 417, 211 (2005).

[19] W. H. Press, Numerical Recipes in Fortran 90 (Cambridge University Press, Cambridge [England] ; New York, 1996), 2nd ed.

[20] B. Kramer and A. MacKinnon, Rep. Prog. Phys. 56, 1469 (1993).

[21] K. Slevin and T. Ohtsuki, Phys. Rev. Lett. 82, 382 (1999).

[22] W. H. Press, B. P. Flannery, S. A. Teukolsky, and W. T. Vetterling, Numerical recipes in FORTRAN : the art of scientific computing (Cambridge University Press, Cambridge, 1992), 2nd ed.

[23] A. G. Galstyan and M. E. Raikh, Phys. Rev. B 56, 1422 (1997).

[24] V. V. Mkhitaryan and M. E. Raikh, Phys. Rev. B 79, 125401 (2009). 